\title{
NORMAL FORM AND ENERGY CONSERVATION OF HIGH FREQUENCY SUBSYSTEMS WITHOUT NONRESONANCE CONDITIONS
}

\author{
DARIO BAMBUSI $(*)$, ANTONIO GIORGILLI $(*)$, \\ SIMONE PALEARI $(*)$, TIZIANO PENATI $(*)$
}

Nota presentata dal s.c. Dario Bambusi

(Adunanza del 28 novembre 2013)

SunTo. Si considera un sistema di oscillatori di frequenza elevata accoppiati con un sistema lento. Si dimostra che l'energia del sottosistema ad alta frequenza varia di poco per tempi molto lunghi. Il risultato qui ottenuto è indipendente dalle relazioni di risonanza tra le alte frequenze. In termini più precisi, si denoti con $\epsilon^{-1}$ la più piccola delle frequenze elevate. Nella prima parte del lavoro si dimostra che l'energia del sistema ad alte frequenze è sostanzialmente conservata per tempi esponenzialmente lunghi con $\epsilon^{-1 / n}$, dove $n$ è il numero di oscillatori ad alta frequenza. A tal fine si fa uso del risultato principale di [1]. Nella seconda parte si dà, in forma completa, una nuova dimostrazione di un risultato analogo valido su tempi dell'ordine di $\epsilon^{-N}$, con $N$ arbitrario. Questo secondo risultato è simile a quello ottenuto nel lavoro [4], che ha ispirato la nota presente.

$* * *$

ABSTRACT. We consider a system in which some high frequency harmonic oscillators are coupled with a slow system. We prove that up to very long times the energy of the high frequency system changes only by a small amount. The result we obtain is completely independent of the resonance relations among the frequencies of the fast system. More in detail, denote by $\epsilon^{-1}$ the smallest high frequency. In the first part of the paper we apply the main result of [1] to prove almost conservation of the energy of the high frequency system over times exponentially long with $\epsilon^{-1 / n}$ ( $n$ being the number of fast oscillators). In the second part of the paper we give a new self-contained proof of a

(*) Dipartimento di Matematica, Università degli studi di Milano, Via Saldini 50, 20133 Milano, Italy

E-mail: dario.bambusi@unimi.it 
similar result which however is valid only over times of order $\epsilon^{-N}$ with an arbitrary $N$. Such a second result is very similar to the main result of the paper [4], which actually was the paper which stimulated our work.

\section{INTRODUCTION}

In the phase space $\mathbb{R}^{2 n} \oplus \mathbb{R}^{2 d} \ni((p, q),(P, Q))$ we consider a Hamiltonian system of the form

$$
H(p, q, P, Q)=h_{\omega}(p, q)+H_{0}(P, Q, q),
$$

where

$$
h_{\omega}(p, q):=\sum_{j=1}^{n} \frac{p_{j}^{2}+\omega_{j}^{2} q_{j}^{2}}{2}
$$

is a system of "fast" harmonic oscillators and $H_{0}$ is an analytic function describing a "slow" system (with canonical variables $P, Q$ ) and its interaction with the fast system. We are interested in the case where the frequencies $\omega_{j}$ are large, so we define

$$
\epsilon:=\frac{1}{\min _{j}\left\{\omega_{j}\right\}},
$$

and study the system in the limit $\epsilon \rightarrow 0$.

In the first part of paper we apply the main result of [1] to prove that $h_{\omega}$ changes by a quantity which is at most of order $\epsilon^{1 / n}$ up to times exponentially long with $\epsilon^{-1 / n}$; in the second part we give a new self contained proof of a stability result very close to a result by Gauckler, Heirer and Lubich [4], which ensures almost invariance of $h_{\omega}$ over times of or$\operatorname{der} \epsilon^{-N}$ with an arbitrary $N$. The main point is that all the results are completely independent of the resonance relations among the frequencies $\omega_{j}$, and thus hold uniformly for all the frequency vectors outside a cube of side $\epsilon^{-1}$.

We recall that systems of the kind of (1) arise in many contexts; here we just mention the problem of the realization of Holonomic constraints, in which the constraints are modeled by very hard springs and one is interested in controlling if the dynamics of the slow system converges, as $\epsilon \rightarrow 0$ to the dynamics of $H_{0}(P, Q, 0)$. This is a very subtle question and 
indeed it is well known that, in general, the convergence of the orbits can occur only for times of order $\epsilon^{-1}$. For longer time scales one can only pursue weaker results, and actually in $[2,3]$ (see also [1]), it has been show that if the frequencies $\omega_{j}$ are either completely resonant or fulfill some Diophantine type inequalities, then $h_{\omega}$ is an approximate integral of motion for times exponentially long with $\epsilon^{-a}$ with $a$ depending on the resonance properties of the frequency vector $\omega$. All the constant involved in the main theorems of $[2,3]$ depend on the properties of $\mathrm{good} / \mathrm{bad}$ approximability of the frequencies by rational vectors.

In their paper [4] Gauckler, Heirer and Lubich used multiscale expansion to show that by restricting attention to time scales of order $\epsilon^{-N}$ with arbitrary $N$, one can find a result independent of the resonance properties of the frequencies, and thus uniform for all frequencies outside an $n$ dimensional hypercube of side $\epsilon^{-1}$. The paper [4] was actually what stimulated the present work.

In the present paper we present two results.

In the first part (Section 2) we look for stability over exponentially long times, in the spirit of Nekhoroshev theory. The novelty of our first result with respect to [3] rests in the uniformity of the constants with respect to small changes in the frequencies $\omega$. Our scheme is reminiscent of that of Lochak in his proof of Nekhoroshev's Theorem [5]: we use Dirichlet approximation theorem in order to approximate the frequencies by completely resonant ones and thus to reduce to a perturbation of a completely resonant system. The error in the approximation by Dirichlet theorem is controlled by a large parameter $\mathrm{Q}$. Then we apply the main theorem of [1] which allows us to put the system in resonant normal form up to a remainder which is exponentially small with an effective small parameter. Some work is required in order to fit into the scheme of [1]. Then one gets a result in which there is an effective small parameter which depends both on $Q$ and on $\epsilon$. So we choose $Q$ as a function of $\epsilon$ in order to minimize the remainder, concluding the proof.

The motivation for the second part of the paper rests in the remark that if one is interested just in power law times, then the result can be obtained by a normal form construction which is purely algebraic (following the original ideas of Birkhoff). The only variant needed with respect to the standard schemes is the one introduced by [4], namely to fix some 
threshold value $\alpha$ for the small denominators and to consider as resonant all the monomials giving rise to small denominators smaller than $\alpha$. Then one can put the system in resonant normal form (in the above sense) up to a remainder of order $\epsilon^{-N}$. Finally, one has to prove that the normal form admits an approximate integral of motion. We prove this last fact using again Dirichlet theorem. We remark that the second result holds also for Hamiltonians which are not analytic but only infinitely differentiable.

Acknowledgments. We thank Christian Lubich for pointing out a mistake in the first version of the paper and for some comments that led to considerable improvements of the paper. This research was funded by the Prin project 2010-2011 "Teorie geometriche e analitiche dei sistemi Hamiltoniani in dimensioni finite e infinite”.

\section{EXPONENTIALLY LONG TIMES}

In the phase space $\mathbb{R}^{2 n} \oplus \mathbb{R}^{2 d} \ni((p, q),(P, Q))$, endowed with the usual euclidean norm, we consider a Hamiltonian system of the form (1) where $H_{0}(P, Q, q)$ is analytic in an open domain of $\mathbb{R}^{2 d+n}$.

We first state the smoothness properties of $H_{0}$ in a precise form. For given $E_{0}$ define the sublevel

$$
\mathcal{S}_{E_{0}}:=\left\{(P, Q) \in \mathbb{R}^{2 d}: H_{0}(P, Q, 0) \leq E_{0}\right\},
$$

and the ball

$$
\mathcal{B}_{\rho}:=\left\{(p, q):\|(p, q)\|^{2}:=\sum_{j} \frac{p_{j}^{2}+q_{j}^{2}}{2} \leq \rho^{2}\right\} .
$$

Remark that $\mathcal{S}_{E_{0}}$ needs not to be compact. Consider the complexification of the phase space and denote by $B(\zeta, R) \subset \mathbb{C}^{2 n+2 d}$ the closed ball of radius $R$ and center $\zeta \equiv(p, q, P, Q)$.

We assume that there exist positive $E_{0}^{*}, E^{*}, R^{*}$ such that, by defining

$$
\mathcal{G}:=\mathcal{B}_{3 \sqrt{E^{*}}} \times \mathcal{S}_{3 E_{0}^{*}}, \quad \mathcal{G}_{R^{*}}^{*}:=\bigcup_{\zeta \in \mathcal{G}} B\left(\zeta, R^{*}\right),
$$


the function $H_{0}$ extends to a bounded analytic function on $\mathcal{G}_{R^{*}}^{*}$, namely to a function fulfilling

$$
\sup _{\mathcal{G}_{R^{*}}^{*}}\left|H_{0}(P, Q, q)\right| \leq C_{H_{0}} .
$$

THEOREM 2.1. Under the above assumptions, there exist positive constants $\epsilon_{*}, C_{1}, C_{2}$ such that, if $0<\epsilon<\epsilon_{*}$ and the initial datum $\left(p^{0}, q^{0}, P^{0}\right.$, $\left.Q^{0}\right)$ fulfills

$$
H_{0}\left(P^{0}, Q^{0}, 0\right) \leq E_{0}^{*}, \quad h_{\omega}\left(p^{0}, q^{0}\right) \leq E^{*},
$$

then along the corresponding solution one bas

$$
\left|h_{\omega}(t)-h_{\omega}(0)\right|<C_{1} \epsilon^{1 / n}, \quad \text { for } \quad|t| \leq C_{2} \exp \left(\frac{\epsilon_{*}}{\epsilon}\right)^{1 / n} .
$$

The constants $\epsilon_{*}, C_{1}, C_{2}$ depend only on $C_{H_{0}}$ and on $n$.

Remark 2.2. The main point is that the constants do not depend on the frequencies and are thus uniform for all frequencies fulfilling (3) with $\epsilon<$ $\epsilon^{*}$.

PROOF. First we remark that by Cauchy inequality for analytic functions one has that the quantities

$$
\left|\frac{\partial H_{0}}{\partial q_{j}}\right|,\left|\frac{\partial H_{0}}{\partial Q_{l}}\right|,\left|\frac{\partial H_{0}}{\partial P_{l}}\right|
$$

are bounded on any domain contained in $\mathcal{G}_{R^{*}}^{*}$, hence the same holds true for the Hamiltonian vector field $X_{H_{0}}$.

To be definite we assume

$$
\min \left\{\omega_{j}\right\}=\omega_{1}=\frac{1}{\epsilon} .
$$

According to Dirichlet theorem, for any $\mathrm{Q}>1$ there exist integers $\mathrm{q} \leq \mathrm{Q}$ and $\left\{\mathrm{p}_{j}\right\}_{j=2}^{n}$ s.t.

$$
\left|\frac{\omega_{j}}{\omega_{1}}-\frac{\mathrm{p}_{j}}{\mathrm{q}}\right| \leq \frac{1}{\mathrm{qQ}^{1 /(n-1)}}, \quad j=2, \ldots, n .
$$


The value of $\mathbf{Q}$ will be fixed later on as a function of $\epsilon$.

Define a new vector of resonant frequencies $\tilde{\omega}$

$$
\tilde{\omega}_{1}:=\omega_{1}, \quad \tilde{\omega}_{j}:=\omega_{1} \frac{\mathrm{p}_{j}}{\mathrm{q}}=\frac{\mathrm{p}_{j}}{\epsilon \mathrm{q}},
$$

and

$$
h_{\tilde{\omega}}:=\sum_{j=1}^{n} \frac{p_{j}^{2}+\tilde{\omega}_{j}^{2} q_{j}^{2}}{2}, \quad h_{1}(q):=\frac{1}{2} \sum_{j=1}^{n}\left(\omega_{j}^{2}-\tilde{\omega}_{j}^{2}\right) q_{j}^{2}, \quad f:=H_{0}+h_{1}
$$

so that the Hamiltonian takes the form

$$
H=h_{\tilde{\omega}}+f,
$$

as required in [1]. Then (12) becomes

$$
\left|\frac{\omega_{j}-\tilde{\omega}_{j}}{\omega_{1}}\right| \leq \frac{1}{\mathrm{qQ}^{1 /(n-1)}}, \quad j=2, \ldots, n .
$$

Furthermore the flow generated by $h_{\tilde{\omega}}$ is periodic with frequency $\omega:=$ $1 / \epsilon \mathbf{q}$.

We redefine the norms and the domains in order to fit the scheme by [1]. So we put

$$
\begin{gathered}
\|\zeta\|_{\sim}^{2} \equiv\|(p, q, P, Q)\|_{\sim}^{2}=\sum_{j=1}^{n} \frac{\left|p_{j}\right|^{2}+\tilde{\omega}_{j}^{2}\left|q_{j}\right|^{2}}{2}+\sum_{l=1}^{d} \frac{\left|P_{l}\right|^{2}+\left|Q_{l}\right|^{2}}{2} \\
\equiv\|(p, q)\|_{\sim}^{2}+\|(P, Q)\|^{2} \\
\quad \tilde{\mathcal{G}}:=\tilde{\mathcal{B}}_{3 \sqrt{E^{*}}} \times \mathcal{S}_{3 E_{0}^{*}}, \quad \tilde{\mathcal{G}}_{\tilde{R}}:=\bigcup_{\zeta \in \tilde{\mathcal{G}}} \tilde{B}(\zeta, \tilde{R})
\end{gathered}
$$

where $\tilde{\mathcal{B}}$ and $\tilde{B}$ are the closed ball in the norm (17).

The relation with the old norms and domains is easily obtained: the new norm (17) is stronger than the euclidean one:

$$
\|q\| \leq \epsilon\|q\|_{\sim},
$$


so, provided $\epsilon$ is small enough and $\mathrm{Q}$ large enough, choosing $\tilde{R}:=R^{*} / 2$, one has $\tilde{\mathcal{G}}_{\tilde{R}} \subset \mathcal{G}_{R^{*}}^{*}$ (strictly and with some finite distance between the boundaries).

We have now to compute the constants involved in the statement of Theorem 4.1 of [1], namely

$\omega_{f}:=\frac{1}{\tilde{R}} \sup _{\zeta \in \tilde{\mathcal{G}}_{\tilde{R}}}\left\|X_{f}(\zeta)\right\|_{\sim} \leq \frac{1}{\tilde{R}}\left[\sup _{\zeta \in \tilde{\mathcal{G}}_{\tilde{R}}}\left\|X_{H_{0}}(\zeta)\right\|_{\sim}+\sup _{\zeta \in \tilde{\mathcal{G}}_{\tilde{R}}}\left\|X_{h_{1}}(\zeta)\right\|_{\sim}\right]$.

Using (10) and (7) one immediately sees that the supremum of $X_{H_{0}}$ is independent of $\epsilon$ and of $\mathbf{Q}$. In order to compute the supremum of $X_{h_{1}}$ recall (16), and remark that

$$
\begin{gathered}
\frac{\omega_{j}}{\tilde{\omega}_{j}}=1+\frac{\omega_{j}-\tilde{\omega}_{j}}{\tilde{\omega}_{j}}, \\
\left|\frac{\tilde{\omega}_{j}-\omega_{j}}{\tilde{\omega}_{j}}\right|=\frac{\left|\frac{\tilde{\omega}_{j}-\omega_{j}}{\tilde{\omega}_{1}}\right|}{\left|\frac{\omega_{j}}{\omega_{1}}-\frac{\omega_{j}-\tilde{\omega}_{j}}{\omega_{1}}\right|} \leq \frac{1 / \mathrm{qQ}^{1 /(n-1)}}{1-\frac{1}{\mathrm{qQ}^{1 /(n-1)}}} \leq \frac{2}{\mathrm{qQ}^{1 /(n-1)}}
\end{gathered}
$$

provided $\mathrm{qQ}^{1 /(n-1)}>2$, from which

$$
\left|\frac{\tilde{\omega}_{j}+\omega_{j}}{\tilde{\omega}_{j}}\right| \leq 3, \quad\left|\frac{\tilde{\omega}_{j}^{2}-\omega_{j}^{2}}{\tilde{\omega}_{j} \omega_{1}}\right| \leq \frac{6}{\mathrm{qQ}^{1 /(n-1)}} .
$$

Thus using (17) and (19) the field $X_{h_{1}}$ admits the upper bound

$$
\begin{aligned}
\left\|X_{h_{1}}(\zeta)\right\|_{\sim}^{2} & =\sum_{j=1}^{n}\left(\omega_{j}^{2}-\tilde{\omega}_{j}^{2}\right)^{2} q_{j}^{2}=\sum_{j=1}^{n} \frac{\left(\omega_{j}^{2}-\tilde{\omega}_{j}^{2}\right)^{2}}{\tilde{\omega}_{j}^{2} \omega_{1}^{2}} \omega_{1}^{2} \tilde{\omega}_{j}^{2} q_{j}^{2} \leq \\
& \leq \frac{1}{\epsilon^{2}}\left[\sup _{j=1, \ldots, n}\left|\frac{\omega_{j}^{2}-\tilde{\omega}_{j}^{2}}{\tilde{\omega}_{j} \omega_{1}}\right|^{2}\right] \sum_{j=1}^{n} \tilde{\omega}_{j}^{2} q_{j}^{2} \\
& \leq\left(\frac{6}{\mathrm{qQ}^{1 /(n-1)}}\right)^{2} \frac{2}{\epsilon^{2}}\|(p, q)\|_{\sim}^{2},
\end{aligned}
$$


which gives

$$
\sup _{\zeta \in \tilde{\mathcal{G}}_{\tilde{R}}}\left\|X_{h_{1}}(\zeta)\right\|_{\sim} \leq\left(\frac{6 \sqrt{2}}{\mathrm{qQ}^{1 /(n-1)}}\right) \frac{\sqrt{9 E^{*}+\tilde{R}^{2}}}{\epsilon} .
$$

So one can put

$$
\omega_{f} \leq C\left[1+\frac{1}{\epsilon \mathrm{qQ}^{1 /(n-1)}}\right],
$$

and the small parameter $\mu$ of Theorem 4.1 turns out to be ${ }^{1}$

$$
\mu:=C \frac{\omega_{f}}{\omega} \leq C \epsilon \mathrm{q}\left(1+\frac{1}{\epsilon \mathrm{qQ}^{1 /(n-1)}}\right) \leq C_{1}\left(\epsilon \mathrm{Q}+\frac{1}{\mathrm{Q}^{1 /(n-1)}}\right) .
$$

Following [1] p. 604, we choose $\mathrm{Q}^{1 /(n-1)}=\epsilon^{-1 / n}$, so that $\epsilon \mathrm{Q}=\epsilon^{1 / n}$ and we can choose $\mu=C_{2} \epsilon^{1 / n}$. Defining $\epsilon_{*}:=C_{2}^{-n}$ and computing the other constants in Theorem 4.1 and its corollaries one gets the thesis.

\section{POWER LAW TIMES}

\subsection{Statement}

The aim of this section is to give an easy proof of a simplified result, in which the control of the energy of high frequency oscillators is obtained only for time scales of order $\epsilon^{-N}$ with an arbitrary $N$. We remark that for the present result $C^{\infty}$ smoothness is enough. Precisely Theorem 3.1 below is true under the assumption that there exists an interval of values of $E_{0}, \rho$ s.t., for any $k$ the $C^{k}$ norm of $H_{0}$ is bounded in $\mathcal{B}_{3 \rho} \times \mathcal{S}_{3 E_{0}}$. Of course, if one fixes a value of $N$ then finite smoothness is also enough.

THEOREM 3.1. Fix a positive (small) $b$, then, for any positive (large) $N$, there exists a positive constant $\epsilon_{*}(N, b)$, such that, if $\epsilon<\epsilon_{*}$, and the initial datum fulfills

$$
E:=h_{\omega}(p, q)<E^{*}, \quad H_{0}(P, Q, 0)<E_{0}^{*},
$$

\footnotetext{
${ }^{1}$ Recall that in Dirichlet Theorem $\mathrm{q} \leq \mathrm{Q}$.
} 
then one has

$$
\left|h_{\omega}(t)-h_{\omega}(0)\right| \leq E b \quad \text { for }|t| \leq \epsilon^{-N} .
$$

Remark 3.2. The constant $\epsilon_{*}$ strongly depends on the dimension $n$ of the fast system, so the result does not extend to infinite dimensional systems. Remark 3.3. In the present statement the change of the energy of the high frequency system is controlled by the parameter $b$, which is arbitrarily small, but independent of $\epsilon$. On the contrary, in the paper [4] one has $b \sim \epsilon^{3 / 4}$.

\subsection{Proof}

We start by preparing the Hamiltonian, subsequently we introduce the kind of expansion needed for the further developments. Then we prove an approximation lemma for the frequencies and finally we prove the normal form lemma that we will use to get Theorem 3.1.

First we scale the variables, the frequencies and the time in a suitable way (see also Sections 2 and 4 of [3]). Together, we introduce the standard complex variables usually needed in order to develop perturbation theory. As in sect. 2 we assume $\omega_{1}=\min \omega_{j}$.

Thus define

$$
\nu_{j}:=\epsilon \omega_{j}, \quad p_{j}=\sqrt{\frac{\nu_{j}}{2 \epsilon}} \frac{\xi_{j}+\eta_{j}}{i}, \quad q_{j}=\sqrt{\frac{\epsilon}{2 \nu_{j}}}\left(\xi_{j}-\eta_{j}\right),
$$

(in particular one has $\nu_{1}=1$ ) so that, by rescaling time to $t^{\prime}:=\epsilon t$, the Hamiltonian of the system (still denoted by $H$ ) takes the form

$$
H=\sum_{j=1}^{n} \nu_{j} \xi_{j} \eta_{j}+\epsilon H_{0}(P, Q, q(\xi, \eta)) .
$$

For the new fast variables $(\eta, \xi)$ we will use the norm

$$
\|(\xi, \eta)\|^{2}=\sum_{j=1}^{n} \nu_{j}\left(\left|\xi_{j}\right|^{2}+\left|\eta_{j}\right|^{2}\right) .
$$


which corresponds to the rescaled energy norm in the original $(p, q)$ variables.

If we define

$$
\rho_{E}:=\sqrt{E},
$$

then $\sum_{j}\left(p_{j}^{2}+\omega_{j}^{2} q_{j}^{2}\right) \leq E$ implies $h_{\nu} \leq \epsilon \rho_{E}^{2}$, which means $(\xi, \eta) \in$ $\mathcal{B}_{\rho_{E} \sqrt{\epsilon}}$. Hence, since the variables $\xi, \eta$ have size of order $\sqrt{\epsilon}$, we have to consider an expansion of the nonlinear terms in both $\sqrt{\epsilon}$ and in $\xi, \eta$. In other words, the scaling (24) introduces two different dependencies on $\sqrt{\epsilon}$ in the Hamiltonian: an implicit one, of size $\mathcal{O}(\sqrt{\epsilon})$ in the scaled variables, and an explicit one in the coefficient in front of any monomial depending on $(\xi, \eta)$, due to dependence on $q$ only.

As anticipated above the main step of the proof consists in putting the system in normal form. We now specify in a precise way what we mean by normal form.

DEFINITION 3.4. Given $\alpha>0$, a monomial $\xi^{l} \eta^{m} \equiv \xi_{1}^{l_{1}} \ldots . \xi_{n}^{l_{n}} \eta_{1}^{m_{1}} \ldots . \eta_{n}^{m_{n}}$ is said to be in $\alpha$-normal form if

$$
|\nu \cdot(l-m)| \leq \alpha .
$$

We are now going to prove that, if $\alpha$ is small enough, then there exists a non vanishing vector $\tilde{\nu}$ such that

$$
h_{\tilde{\nu}}(\xi, \eta):=\sum_{j=1}^{n} \tilde{\nu}_{j} \xi_{j} \eta_{j}
$$

Poisson commutes with all the monomials in normal form.

LEMMA 3.5. Fix $N>0$, then there exists a non negative sequence $\left\{\alpha_{i}\right\}_{i \geq 1}$, with $\lim _{i \rightarrow \infty} \alpha_{i}=0$, such that, for every frequency vector $\nu$ there exists a new frequency vector $\tilde{\nu}$, depending on $\alpha_{i}$, which fulfills

$$
\begin{gathered}
\sup _{j=1, \ldots, n}\left|\tilde{\nu}_{j}-\nu_{j}\right| \leq \frac{\alpha_{i}}{N}, \\
\left\{h_{\tilde{\nu}} ; \xi^{l} \eta^{m}\right\}=0,
\end{gathered}
$$

for all monomials $\xi^{l} \eta^{m}$ in $\alpha_{i}$ normal form satisfying $|l|+|m| \leq N$. 
PROOF. We use again Dirichlet theorem. The form we choose is the one according to which, for any $\nu \in \mathbb{R}^{n-1}$ the inequalities

$$
\left|\nu_{j}-\frac{\mathrm{p}_{j}}{\mathrm{q}}\right| \leq \frac{1}{\mathrm{q}^{1+1 /(n-1)}}, \quad j=2, \ldots, n
$$

have infinitely many solutions $\mathrm{q} \in \mathbb{N}, \mathrm{p}_{j}(\mathrm{q}) \in \mathbb{Z}$. In particular the q's form a diverging sequence $\mathrm{q}_{i}$. We identify the sequence $\mathrm{q}_{i}$ with the corresponding value of $\mathrm{q}$ (instead of using $i$ ). Define $\tilde{\nu}_{j}:=\mathrm{p}_{j} / \mathrm{q}, j=$ $2, \ldots, n-1, \tilde{\nu}_{1}:=\nu_{1}=1$ and $\alpha_{\mathrm{q}}(N, n):=N / \mathrm{q}^{1+1 /(n-1)}$. We are now going to prove that $\tilde{\nu} \cdot k \neq 0$ with $|k| \leq N$ implies $|\nu \cdot k|>\alpha_{\mathrm{q}}$. First remark that $\tilde{\nu} \cdot k \neq 0$ implies $|\tilde{\nu} \cdot k| \geq 1 / \mathrm{q}$ (since $\tilde{\nu}_{j}$ are rationals), so that one has

$$
\begin{aligned}
|\nu \cdot k| & \geq|\tilde{\nu} \cdot k|-|(\nu-\tilde{\nu}) \cdot k| \geq \frac{1}{\mathrm{q}}-|\tilde{\nu}-\nu||k| \geq \\
& \geq \frac{1}{\mathrm{q}}-\frac{N}{\mathrm{q}^{1+1 /(n-1)}}=\frac{1}{\mathrm{q}}-\alpha_{\mathrm{q}}=\alpha_{\mathrm{q}}\left[\frac{1}{\left(N^{n-1} \alpha_{\mathrm{q}}\right)^{\frac{1}{n}}}-1\right],
\end{aligned}
$$

but, provided $\alpha_{\mathrm{q}}$ is small enough with respect to $N^{n-1}$ the square bracket is bigger than 1 and the thesis follows.

We fix now once for all $\alpha$ as

$$
\alpha \equiv \alpha_{i} \leq \frac{b N}{21} .
$$

In the following we are going to construct a canonical transformation which puts the Hamiltonian in normal form up to order $(\sqrt{\epsilon})^{2 N}=$ $\epsilon^{N}$. We first introduce the class of polynomials that we will meet in the construction and the degree that we will assign to each of them.

DEFINITION 3.6. Let $\mathcal{U} \subset \mathcal{S}_{3 E_{0}^{*}}$ be an open domain. For $s \geq 0$, the space $\mathcal{P}_{s} \equiv \mathcal{P}_{s}(\mathcal{U})$ is the space of the linear combinations, with coefficients in $C^{\infty}(\mathcal{U})$, of the monomials of the form

$$
(\sqrt{\epsilon})^{a+2} \xi^{l} \eta^{m}
$$


where the indexes fulfill the conditions

$$
a+|l|+|m|=s, \quad a \geq|l|+|m| .
$$

If $g \in \mathcal{P}_{s}$, then the index $s$ will be called the order of the polynomial $g$.

In the following, when not needed, we will not specify the domain $\mathcal{U}$. It is immediate to verify the following Lemma

LEMMA 3.7. Let $g_{1} \in \mathcal{P}_{s_{1}}$ and $g_{2} \in \mathcal{P}_{s_{2}}$, then

$$
\left\{g_{1} ; g_{2}\right\} \in \mathcal{P}_{s_{1}+s_{2}} \oplus \mathcal{P}_{s_{1}+s_{2}+2} .
$$

PROOF. Indeed

$$
\left\{g_{1} ; g_{2}\right\}=\left\{g_{1} ; g_{2}\right\}_{P, Q}+\left\{g_{1} ; g_{2}\right\}_{\xi, \eta} ;
$$

the first term at r.h.s. belongs to $\mathcal{P}_{s_{1}+s_{2}+2}$ and the second one belongs to $\mathcal{P}_{s_{1}+s_{2}}$.

Remark 3.8. Consider $\left\{h_{\nu} ; g\right\}$, with $g \in \mathcal{P}_{s}$ and $s \geq 1$. In this case it, due to the lack of a prefactor $\epsilon$ in front of $h_{\nu}$, is immediate to verify that $\left\{h_{\nu} ; g\right\} \in \mathcal{P}_{s}$.

Remark 3.9. Moreover, it is useful to stress that both in case of Lemma 3.7 and in the case of $\left\{h_{\nu} ; g\right\}$, the parity of the space $\mathcal{P}_{s}$ is preserved by the Poisson brackets. Due to the structure of the perturbation $\epsilon H_{0}$, we will deal only with even parity spaces $\mathcal{P}_{2 s}$.

It is useful to extend the definition to functions of $\xi, \eta, \sqrt{\epsilon}$ of class $C^{\infty}$ and to introduce the space of the functions that will play the role of remainders.

DEFINITION 3.10. Let $F((P, Q),(\xi, \eta), \sqrt{\epsilon}), F \in C^{\infty}\left(\mathcal{U} \times \mathcal{B}_{\rho} \times \mathcal{B}_{\sqrt{\epsilon^{\sharp}}}\right)$ for some positive $\rho, \sqrt{\epsilon^{\sharp}}$. We say that $F \in \overline{\mathcal{P}}(\mathcal{U})$ if each of its Taylor polynomials in $\xi, \eta, \sqrt{\epsilon}$ belongs to some of the spaces $\mathcal{P}_{s}(\mathcal{U})$.

Given a function $F \in \overline{\mathcal{P}}$ we can define the projector $\Pi_{s}$ which extracts from $F$ its component in $\mathcal{P}_{s}$. 
DEFINITION 3.11. A function $F \in \overline{\mathcal{P}}(\mathcal{U})$ will be said to belong to $\mathcal{R}_{r}(\mathcal{U})$ if one has $\Pi_{s} F=0, \forall s \leq r$.

Remark 3.12. For any $N$ one can expand $H_{0}$ in Taylor series in the variables $\xi, \eta$ at order $N$, getting

$\epsilon H_{0}=\sum_{s=0}^{N} f_{s}+R^{(N)}, \quad f_{s}(P, Q, \xi, \eta)=\epsilon \sum_{|l|+|m|=s} a_{l m}(P, Q) \xi^{l} \eta^{m} \epsilon^{s / 2}$

and $R^{(N)}$ having a zero of order $N+1$ in the variables $\xi, \eta$. Thus one has $f_{s} \in \mathcal{P}_{2 s}$ and $R^{(N)} \in \mathcal{R}_{2 N+1}$ (see Remark 3.9).

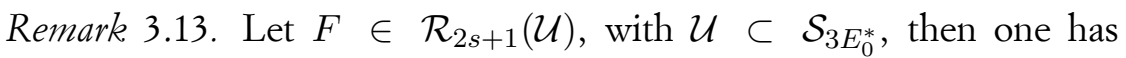
$\sup _{\mathcal{U} \times B_{\rho \sqrt{\epsilon}}}|F| \leq C \sqrt{\epsilon}^{2 s+3}$. The constant depends in particular on $\mathcal{U}$ and on $\rho$. Similar inequalities hold for the derivatives of $F$.

The normalizing transformation will be constructed using the Lie transform $\phi_{\chi}$, namely the time one flow of an auxiliary Hamiltonian $\chi \in \mathcal{P}_{2 r}$ with $r \geq 1$. The main properties of the Lie transform are summarized in the next lemma.

LEMMA 3.14. Let $\mathcal{S}_{3 E_{0}^{*}} \supset \mathcal{U}_{1} \supset \mathcal{U}_{2} \supset \mathcal{U}_{3} \supset \mathcal{S}_{2 E_{0}^{*}}$ be open sets (the inclusion must be strict) and let $3 \rho_{E}>\rho_{1}>\rho_{2}>\rho_{3}>2 \rho_{E}$ be positive parameters. Let $\chi \in \mathcal{P}_{s}\left(\mathcal{U}_{1}\right)$ with $s \geq 1$. Then there exists $\epsilon_{\sharp}$, such that, if $\epsilon<\epsilon_{\sharp}$, then one bas

$$
\mathcal{U}_{1} \times B_{\rho_{1} \sqrt{\epsilon}} \supset \phi_{\chi}\left(\mathcal{U}_{2} \times B_{\rho_{2} \sqrt{\epsilon}}\right) \supset \mathcal{U}_{3} \times B_{\rho_{3} \sqrt{\epsilon}} .
$$

The constant $\epsilon_{\sharp}$ depends only on the above sets $\mathcal{U}_{i}$, on $\rho_{i}$ and on the norm $\mathcal{C}^{1}\left(\mathcal{U}_{1}\right)$ of the coefficients of the development of $\chi$ in $\xi, \eta, \sqrt{\epsilon}$.

Let $F \in \mathcal{P}_{r}$, then one has

$$
\left[F \circ \phi_{\chi}-F\right] \in \mathcal{R}_{s+r}
$$

and

$$
\left[h_{\nu} \circ \phi_{\chi}-\left(h_{\nu}+\left\{\chi ; h_{\nu}\right\}\right)\right] \in \mathcal{R}_{2 s-1} .
$$


PROOF. The statement on the existence of the flow and the way it transforms open domains immediately follows from the standard theory of existence and uniqueness of ODEs.

To get (38) and (39) one uses

$$
\frac{\mathrm{d}}{\mathrm{d} t} F \circ \phi_{\chi}^{t}=\{\chi ; F\} \circ \phi_{\chi}^{t},
$$

from which,

$$
F \circ \phi_{\chi}=F+\{\chi ; F\}+\int_{0}^{1}(1-s)\{\chi ;\{\chi ; F\}\} \circ \phi_{\chi_{\chi}^{s}} \mathrm{~d} s,
$$

which holds both for the case of the function $F$ of the statement and for the function $h_{\nu}$. Then using Lemma 3.7, the fact that $\left\{\chi ; h_{\nu}\right\} \in \mathcal{P}_{s}\left(\mathcal{U}_{1}\right)$ (see Remark 3.8) and standard estimates the thesis follows.

We are now ready to state and prove the iterative lemma which yields the existence of the normal form.

LEMMA 3.15. There exists a sequence of domains $\mathcal{S}_{3 E_{0}^{*}} \supset \mathcal{U}_{0} \supset \mathcal{U}_{1} \supset$ $\ldots \supset \mathcal{U}_{N+1} \supset \mathcal{S}_{2 E_{0}^{*}}$ and a sequence of positive parameters $3 \rho_{E}>\rho_{0}>$ $\rho_{1}>\ldots>\rho_{N+1}>2 \rho_{E}$ with the following property: for any $1 \leq r \leq N$ there exists a positive $\epsilon_{r}$, such that, if $\epsilon<\epsilon_{r}$ then there exists a canonical transformation $T^{(r)}: \mathcal{U}_{r} \times B_{\rho_{r} \sqrt{\epsilon}} \rightarrow \mathcal{U}_{0} \times B_{\rho_{0} \sqrt{\epsilon}}, T^{(r)}\left(\mathcal{U}_{r} \times B_{\rho_{r} \sqrt{\epsilon}}\right) \supset$ $\mathcal{U}_{r+1} \times B_{\rho_{r+1} \sqrt{\epsilon}}$ such that $H \circ T^{(r)}$ is in normal form at order $2 r$, namely $\forall l \leq 2 r$ the polynomial $\Pi_{l}\left[H \circ T^{(r)}\right]$ is in normal form. One also has

$$
\left[h_{\widetilde{\nu}} \circ T^{(r)}-h_{\widetilde{\nu}}\right] \in \mathcal{R}_{1}, \quad\left[\epsilon H_{0} \circ T^{(r)}-\epsilon H_{0}\right] \in \mathcal{R}_{3} .
$$

The sets $\mathcal{U}_{j}$, as well as the parameters $\rho_{j}$, the $C^{k}$ norm of $T^{(r)}$, and the quantity $\epsilon_{r}$, depend on the frequency only through the parameter $\alpha$. Finally the transformed Hamiltonian contains only terms of even order (in the sense of definition 3.6).

PROOF. The proof follows the standard proof of Birkhoff normal form theorem. The theorem is true for $r=0$. We assume it for $r$ and prove it for $r+1$. We construct the transformation increasing by one the order of the non normalized part of the Hamiltonian as the Lie transform 
generated by a function $\chi_{r+1} \in \mathcal{P}_{2(r+1)}$. First remark that, according to (38), (39) the transformed Hamiltonian is automatically in normal form at order $2 r$. We are going to choose $\chi_{r+1}$ in such a way that

$$
\Pi_{2(r+1)}\left[H \circ T^{(r)} \circ \phi_{\chi_{r+1}}\right] \equiv\left\{\chi_{r+1} ; h_{\nu}\right\}+\Pi_{2(r+1)}\left[H \circ T^{(r)}\right]
$$

is in normal form too. To this end write

$$
\Pi_{2(r+1)}\left[H \circ T^{(r)}\right]=\sum_{a, l, m} P_{a, l, m}(P, Q) \sqrt{\epsilon}^{a+2} \xi^{l} \eta^{m},
$$

where the indexes fulfill the limitations $a+|l|+|m|=2(r+1)$ and $a \geq|l|+|m|$, so that, in particular $|l|+|m| \leq r+1 \leq N$. Define now

$$
\chi_{r+1}:=\sum_{(l, m) \in N R, a} \frac{P_{a, l, m}(P, Q)}{\mathrm{i} \nu \cdot(l-m)} \sqrt{\epsilon}^{a+2} \xi^{l} \eta^{m}
$$

where the nonresonant set $N R$ is defined by

$$
N R:=\{(l, m):|\nu \cdot(l-m)|>\alpha\} .
$$

Then, the $C^{k}$ norm of $\chi_{r+1}$ is controlled by the $C^{k}$ norm of $\Pi_{2(r+1)}[H \circ$ $\left.T^{(r)}\right]$ divided by $\alpha$, and therefore the statement on the $C^{k}$ norm of the transformation holds. The statement on the domain of definition of the transformation follows from $\mathcal{U}_{r+2} \subset \phi_{\chi_{r+1}}\left(\mathcal{U}_{r+1}\right) \subset \mathcal{U}_{r}$ which is consequence of Lemma 3.14. The same is true for (41) (which at leading order follows directly from Lemma 3.7) and the statement on the dependence of the parameters on the frequency.

END OF THE PROOF OF THEOREM 3.1. Consider $T^{(N)}$ and denote the new variables by $\left(P^{\prime}, Q^{\prime}, \xi^{\prime}, \eta^{\prime}\right)$, namely $(P, Q, \xi, \eta)=T^{(N)}\left(P^{\prime}, Q^{\prime}\right.$, $\left.\xi^{\prime}, \eta^{\prime}\right)$ and by $h_{\widetilde{\nu}}^{\prime}:=\sum \widetilde{\nu}_{j} \xi_{j}^{\prime} \eta_{j}^{\prime}$. Compute

$$
\begin{aligned}
\left|h_{\nu}(t)-h_{\nu}(0)\right| \leq & \left|h_{\nu}(t)-h_{\widetilde{\nu}}(t)\right|+\left|h_{\widetilde{\nu}}(t)-h_{\widetilde{\nu}}^{\prime}(t)\right| \\
& +\left|h_{\widetilde{\nu}}^{\prime}(t)-h_{\widetilde{\nu}}^{\prime}(0)\right|+\left|h_{\widetilde{\nu}}^{\prime}(0)-h_{\widetilde{\nu}}(0)\right|+\left|h_{\widetilde{\nu}}(0)-h_{\nu}(0)\right| .
\end{aligned}
$$


Assume for a while that $\left\|\left(\xi^{\prime}(t), \eta^{\prime}(t)\right)\right\| \leq 2 \rho_{E} \sqrt{\epsilon}$ for $|t| \leq \epsilon^{-N}$, then from Lemma $3.15\|(\xi(t), \eta(t))\| \leq 3 \rho_{E} \sqrt{\epsilon}$ and one can use (30) and (41) to estimate the different terms of (44) and (45) by

$$
4 \rho_{E}^{2} \epsilon \frac{\alpha}{N}+C \epsilon^{2}+\left|\left\{h_{\widetilde{\nu}}, H \circ T^{(N)}\right\}\right||t|+C \epsilon^{2}+9 \rho_{E}^{2} \epsilon \frac{\alpha}{N} .
$$

Indeed from $h_{\widetilde{\nu}}-h_{\widetilde{\nu}}^{\prime} \in \mathcal{R}_{1}$ it follows immediately $\left|h_{\widetilde{\nu}}(t)-h_{\widetilde{\nu}}^{\prime}(t)\right|<C \epsilon^{2}$. On the other hand, one has to recall that $h_{\nu}$ is the norm (see (26)) and that $h_{\widetilde{\nu}}$ is close to $h_{\nu}$ because of (30)

$$
\left|h_{\nu}-h_{\widetilde{\nu}}\right| \leq\left(\sup _{j=1, \ldots, n}\left|\nu_{j}-\tilde{\nu}_{j}\right|\right) \sum_{j}\left(\left|\xi_{j}\right|^{2}+\left|\eta_{j}\right|^{2}\right) \leq \frac{\alpha}{N}\|(\xi, \eta)\|^{2} .
$$

Now, since $H \circ T^{(N)}$ is in normal form, one has $\left\{h_{\widetilde{\nu}}, H \circ T^{(N)}\right\} \in$ $\mathcal{R}_{2 N+1}$ which in turn implies $\left|\left\{h_{\widetilde{\nu}}, H \circ T^{(N)}\right\}\right| \leq C \epsilon^{N+2}$ and therefore, for the considered times the third term is smaller than $C \epsilon^{2}$.

Take now $\epsilon$ so small that the sum of the second, the third and the fourth term of (44),(45) does not exceed $\alpha \rho_{E}^{2} \epsilon / N$, then going back to the original variables and recalling that, from (33), $\alpha \leq N b / 21$ the estimate (23) follows.

We still have to prove that for $|t| \leq \epsilon^{-N}$ all the variables are in the domain of validity of the normal form. Concerning the fast variables this is a consequence of an argument similar to that of Lyapunov's theorem which gives

$$
h_{\widetilde{\nu}}^{\prime}(t) \leq h_{\widetilde{\nu}}^{\prime}(0)+\left|h_{\widetilde{\nu}}^{\prime}(t)-h_{\widetilde{\nu}}^{\prime}(0)\right| \leq \rho_{E}^{2} \epsilon(1+\alpha)+C \epsilon^{2} \leq 2 \rho_{E}^{2} \epsilon .
$$

Concerning the variables $\left(P^{\prime}, Q^{\prime}\right)$ we exploit the conservation of the Hamiltonian. To this end denote $\hat{h}(P, Q):=H_{0}(P, Q, 0)$ and $H_{P}:=$ $H_{0}-\hat{h}$, and remark that $\left|H_{P}\right|<C \epsilon$, so that one has (in the $(P, Q)$ variables)

$$
\hat{h}(t)=\hat{h}(0)+h_{\omega}(0)-h_{\omega}(t)+H_{P}(0)-H_{P}(t)
$$


so that, recalling the second of (33), one has

$$
\hat{h}(t) \leq E_{0}^{*}+E b+C \epsilon<\frac{3}{2} E_{0}^{*},
$$

provided $b$ and $\epsilon$ are small enough. It follows that $\hat{h}^{\prime}(t) \leq 2 E_{0}^{*}$ on the considered time scale. The result then holds in the rescaled time. To get the result in the physical time, just repeat the whole argument with $N+1$ in place of $N$.

\section{REFERENCES}

[1] Dario Bambusi and Antonio Giorgilli, Exponential stability of states close to resonance in infinite-dimensional Hamiltonian systems, J. Statist. Phys. 71 (1993), no. 3-4, 569-606.

[2] Giancarlo Benettin, Luigi Galgani, and Antonio Giorgilli, Realization of holonomic constraints and freezing of high frequency degrees of freedom in the light of classical perturbation theory. I, Comm. Math. Phys. 113 (1987), no. 1, 87-103.

[3] Giancarlo Benettin, Luigi Galgani, and Antonio Giorgilli, Realization of holonomic constraints and freezing of high frequency degrees of freedom in the light of classical perturbation theory. II, Comm. Math. Phys. 121 (1989), no. 4, 557-601.

[4] Ludwig Gauckler, Ernst Hairer, and Christian Lubich, Energy separation in oscillatory Hamiltonian systems without any nonresonance condition, Comm. Math. Phys. 321 (2013), no. 3, 803815.

[5] Pierre Lochak, Canonical perturbation theory: an approach based on joint approximations, Uspekhi Mat. Nauk 47 (1992), no. 6(288), 59-140. 